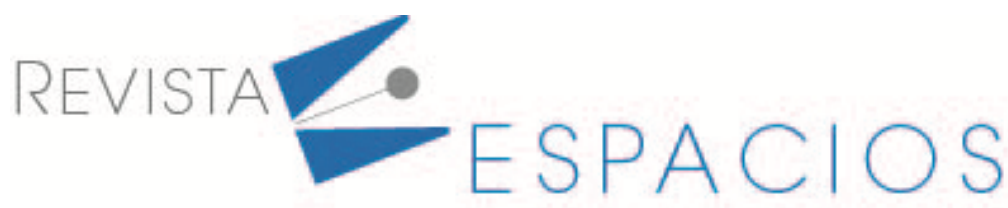

Vol. 41 (50) $2020 \cdot$ Art. 9

\title{
Análisis de las falencias del sector apicultor en la provincia del Guayas (Ecuador)
}

\section{Analysis of the failures of the beekeeping sector in the province of Guayas}

\author{
ROSILLO Toro, W. ${ }^{1}$ \\ VIVANCO Hidalgo, I. ${ }^{2}$ \\ REYES Muñoz, E. ${ }^{3}$ \\ RODRIGUEZ Robles, D. 4
}

\section{Resumen}

El sector de la miel de abeja en otros países es muy dinámico y desarrollado, pero en el Ecuador es otra la realidad, en especial en la provincia del Guayas que, aunque es una provincia apta para la apicultura no está siendo explotada de manera correcta causando así un desabastecimiento en el mercado el cual es cubierto con importaciones provenientes de China, México, Perú y Argentina, otro principal problema es el número total de mieles adulteradas que en algunos casos son difíciles de identificar por el consumidor. En el presente trabajo se hace un análisis del sector (producción. comercialización, marcas, precios, producto de origen) para así dar una mayor compresión al lector de la realidad por la cual atraviesa el sector apicultor.

Palabras clave: apicultura, miel, producción, comercialización, consumidor.

\begin{abstract}
The bee honey sector in other countries is a very dynamic and developed sector, but in Ecuador the reality is different, especially in the province of Guayas which, although it is a province for beekeeping, is not being exploited correct causing a shortage in the market which is covered with imports from China, Mexico, Peru and Argentina, another main problem is the total number of adulterated honeys that in some cases are difficult to identify by the consumer. In the resent work, an attempt will be made to analyze the sector (production, marketing, brands, prices, original product) in order to give the reader a greater understanding of the reality that the beekeeping sector is going through.

key words: beekeeping, honey, production, marketing, consumer.
\end{abstract}

\footnotetext{
${ }^{1}$ Docente Universitario. Departamento de Vinculación. Universidad de Guayaquil. willian.rosilloto@ug.edu.ec

2 Docente Universitario. Departamento de Vinculación. Universidad de Guayaquil. isauro.vivancoh@ug.edu.ec

${ }^{3}$ Estudiante Universitario. Proceso de Titulacion Universidad de Guayaquil. eduardo.reyesmu@ug.edu.ec

${ }^{4}$ Estudiante Universitario. Proceso de Titulacion. Universidad de Guayaquil. Denisse.rodriguezr@ug.edu.ec
} 


\section{Introducción}

La apicultura en el país se ha practicado desde la época colonial. Esta actividad solo ha sido destinada al autoconsumo y este sector no ha sido considerado como un sector generador de ingresos y no se ha desarrollo debidamente.

El Ecuador cuenta con condiciones climáticas apropiadas para el asentamiento y reproducción de apiarios, razón por la cual la actividad apícola es realizada en diversas provincias del país, entre estas la provincia del Guayas. No obstante, la misma se ha venido desarrollando a baja escala lo cual ha ocasionando un retraso en el desarrollo del sector y un desaprovechamiento de la apicultura. Esto debido a una precaria industrialización de los modos de producción y del desarrollo de nuevas tecnologías para mejorar la efectividad por colmena y los procesos productivos apícolas.

El presente trabajo tiene como objetivo analizar y describir las principales problemáticas que aquejan el sector apicultor de la provincia del Guayas, basado en el estudio de la estructura productiva y comercial de la actividad apícola, mediante instrumentos de investigación tales como entrevistas y encuestas que permitirán obtener los resultados y conclusiones respectivas.

\subsection{La apicultura en el mundo}

"Actualmente la apicultura es una actividad que se ha venido desarrollando en todos los países del mundo debido a su gran adaptabilidad a diferentes climas. Efectivamente puede haber esta actividad apícola desde $80 \mathrm{~m}$ hasta $3400 \mathrm{~m}$ sobre el nivel del mar. (Granda, 2017)

China es un país que es completamente consciente de la importancia que tiene la apicultura y específicamente la polinización que brindan las abejas como ayuda a la producción agrícola. En el año 2008 China invirtió cerca de 31 millones de dólares en investigaciones sobre la producción de la miel de abeja y los beneficios de la polinización. En la actualidad China es el mayor productor de la miel de abeja a nivel mundial con 543.000 toneladas para el año 2019. .

México es otro país que le presta atención a la producción de la miel de abeja, en estados como Yucatán, Quintana Roo y Campeche, que producen cerca del $60 \%$ de la producción total de México. (Magaña, Cortés, Barrientos, \& García, 2016). En el año 2019 México produjo alrededor de 51.070 toneladas de las cuales el 78,5\% se usó en el mercado externo.

\subsection{La apicultura en Ecuador}

En Ecuador se desconoce cuando inicio la actividad apícola, sin embargo se sabe que por mucho tiempo los indígenas se dedicaron al cuidado de las abejas a pequeña escala. En el siglo XIX se dio un importante paso al desarrollo de la apicultura, gracias a misiones de religiosos que iniciaron la actividad apícola en conventos en la ciudad de Cuenca para satisfacer la demanda interna de miel.

El consumo de miel de abeja se hizo famoso en la ciudad, como un endulzante natural, y por los beneficios médicos que proveía, la apicultura se consolidó como una actividad económicas de la ciudad de Cuenca.

Gracias a las características ambientales, geológicas y florísticas de esta ciudad, la apicultura se logró afianzar ayudando a la adaptación de colmenas europeas en Ecuador. Esto permitió que la apicultura se diseminara rápidamente por todo el territorio nacional con las primeras apícolas privadas en Guayaquil y mas tarde en Loja, Manabí y Quito. 
"El auge de la apicultura comienza en el año 1993, con un total de 38.500 colmenas." (Granda, 2017). Así mismo este autor indica: Para el año 2004 en Zamora, Loja y El Oro, es decir la zona sur del Ecuador. se registraron 51 especies de abejas. ,

En la actualidad la apicultura es una actividad importante en regiones de la sierra, debido a su amplia flora melífera, En el año 2014 se registraron un total de 12.188 tonelatas de miel en 902 explotaciones de las cuales el $22 \%$ en Pichincha, lo que confirma que el $70 \%$ de la producción apícola se da en la sierra, mientras que el $23 \%$ se da en la costa y solo un $7 \%$ en el Oriente. (Agrocalidad, 2014)

La miel de abeja es el producto que mayor representación tiene en la apicultura ecuatoriana con un 85\%, la cera de abeja se produce en un $5 \%$, polen en un $3 \%$, propóleo $6 \%$, jalea real $1 \%$, y Apitoxina $0.1 \%$. El $100 \%$ de las explotaciones apícolas aproximadamente el $90 \%$ ocurre en sectores rurales es propicio para la agricultura y el $10 \%$ restante en zonas urbanas. (Granda, 2017)

\subsection{La apicultura en la provincia del Guayas}

Las provincias que mayor ingreso aportan al sector apícola son, la provincia de Chimborazo y la provincia del Pichincha, seguida por la provincia del Guayas, otras con menor desempeño como Loja, y Zamora Chinchipe.

\section{Metodología}

Para poder realizar un análisis del sector apícola se efectuó una investigación de carácter descriptivo y exploratorio que consiste en una revisión de documentos y fuentes oficiales, la recolección de datos, por medio de encuestas dirigidas a apicultores y a consumidores, y un estudio de mercado. El carácter exploratorio radica en que en la actualidad no existe una base de datos que arroje cifras reales, tanto en la producción como en la comercialización de la miel de abeja en la provincia del Guayas.

Las encuestas fueron dirigidas a 32 apicultores y 384 consumidores de la provincia del Guayas. que se encuentran inscritos en el registro nacional sanitario del MAGAP 2018.

\section{Resultados}

A continuación, se describen las principales falencias que presenta el sector de la apicultura, que provienen de los resultados obtenidos de los instrumentos de investigación y permiten proyectar una mejor perspectiva de la situación actual del sector.

\subsection{Problema con el registro de apicultores en el MAGAP}

En la provincia del Guayas existen 25 cantones, que se dedican a la actividad apícola de estos según el MAGAP solo 16 cantones tienen presencia de esta actividad quedando 9 cantones sin presencia apícola.

Que no exista presencia apícola en el registro del Magap, (Ministerio de Agricultura, Ganadería, Acuacultura y Pezca, significa que no hay apicultores registrados en el catastro que manejan los organismos oficiales. Pero esto no significa que no haya apicultores en estos lugares. Según el Ing. José Baidal, apicultor aficionado y de profesión vía entrevista supo expresar que los registros que posee el MAGAP son solo, de aquellos apicultores que piden asesoramiento a la institución o aquellos que necesitan ayudas económicas. Es decir, se quedan fuera de este catastro muchos apicultores de la provincia del Guayas lo cual disminuye las dimensiones de la capacidad productiva y del recurso humano con el que verdaderamente se cuenta.

Según el catastro del 2018 del MAGAP existen solo 43 apicultores esparcidos por toda la provincia, pero se estima que son cerca de 120 . 
Tabla 1

Apicultores por tamaño en la provincia del Guayas

\begin{tabular}{|c|c|c|c|}
\hline Apicultores por tamaño & $\begin{array}{l}\text { Numero de } \\
\text { apicultores }\end{array}$ & Numero de colmenas & $\begin{array}{l}\% \text { de colmena } x \\
\text { tipo de apicultor }\end{array}$ \\
\hline Pequeños (1-50 colmenas) & 41 & 570 & 78,30 \\
\hline Medianos (51 a 150 colmenas) & 2 & 158 & 21,70 \\
\hline Grandes (más de colmenas) & 0 & 0 & \\
\hline TOTAL & 43 & 728 & $100 \%$ \\
\hline
\end{tabular}

Como se puede observar en la tabla 1 los pequeños apicultores son 78,30\% los medianos apicultores representan el restante es decir el $21,70 \%$. Hay que aclarar que en el territorio no existe presencia de los grandes apicultores.

\subsection{Falta de tecnificación en los procesos productivos de la miel de abeja}

Otro de los problemas que afecta al sector es la falta de tecnología para la extracción, el envasado y etiquetado del producto. Esto se pudo notar en la encuesta realizada a los apicultores y puede ser debida al costo de la maquinaria y equipos que los pequeños y medianos apicultores no pueden pagar. Ellos proceden en forma artesanal, con extractores improvisados, mesas de madera em hechos a base de materiales que no son los correctos así como mesas de madera y tinas de plástico donde es fácil que las bacterias proliferen y dañan la miel, Según el manual de buenas prácticas apícolas lo correcto es utilizar utensilios de acero inoxidable. Gran parte de los apicultores realizan de esta manera errónea su actividad debido al desconocimiento y esto trae como consecuencia una pérdida de calidad de sus productos y que sea más complicado obtener una certificación sanitaria para poderlos comercializar de una mejor manera.

Figura 1

Porcentaje de apicultores

tecnific ados y no tecnificados

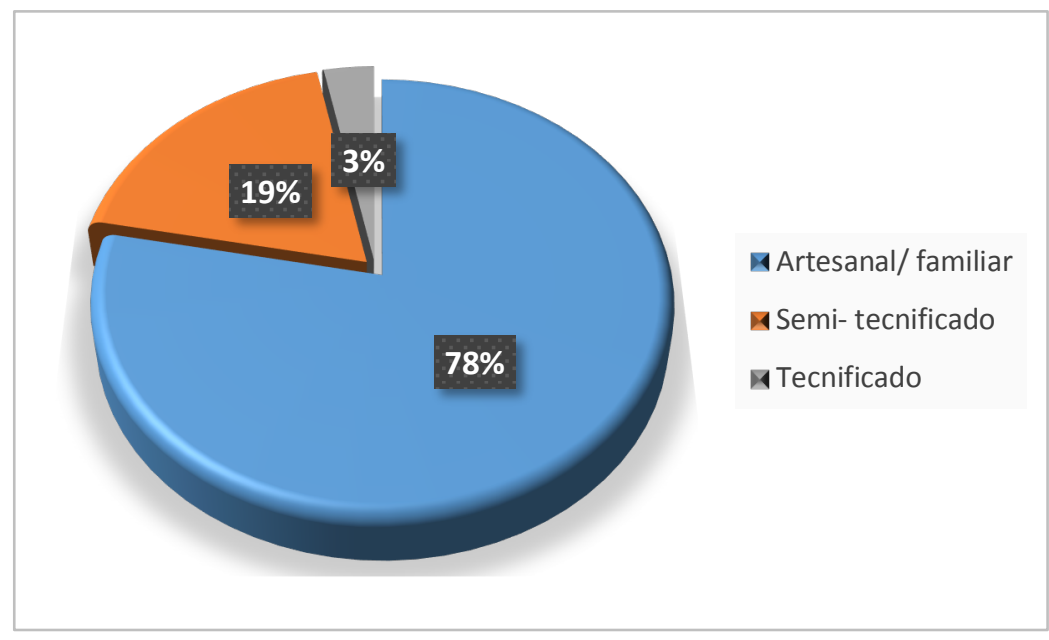

Fuente. Los autores

Como se puede observar en la figura 1 el $78 \%$ de los apicultores en la provincia son apicultores artesanales, el 19\% semi-tecnificados y solo el 3\% tecnificado. Para poder hacer esta aseveración se procedió a verificar cuáles son los utensilios utilizados en la apicultura y que curso habían realizado para familiarizarse con este tipo de trabajo. Es importante mencionar que un curso completo para un apicultor principiante consta de 2 horas al día durante 6 meses y una carga horaria de 312 horas esto y un precio promedio de \$400 dólares" (Ivars, 2015). 
Este tipo de cursos son importantes para los apicultores ya que se les enseña a cómo hacer frente a de las abejas tales como: "Acarapisosis de las abejas melíferas, Loque americana de las abejas melíferas, Loque europea de las abejas melíferas, Infestación por el escarabajo de las colmenas, Infestación de las abejas melíferas por el ácaro, Varroosis de las abejas melíferas." (OIE, s.f.)

Todas estas enfermedades son las principales reconocidas por OIE (Organización Mundial de Sanidad Animal) siendo la varoosis la principal causante de la muerte de colonias enteras. Igualmente otras amenazas como el cambio climático o las sequias o el uso indiscriminado de plaguicidas afectan la salud de las abejas.

La mayoría de los apicultores no está lista para hacer frente a estas amenazas y sus colmenas sucumben sufren serias consecuencia. Algunos productores se ven forzados a auto educarse y cuando se presenta alguna enfermedad la experiencia y la solidaridad entre apicultores prima ante la escasez de técnicos preparados en el sector para recibir un verdadero asesoramiento.

EI MAGAP suele realizar charlas de dos horas de duración pero estas no son continuas y no se hace un seguimiento de los conocimientos adquiridos por los apicultores lo cual se nota a la hora de poner en práctica los conocimientos supuestamente adquiridos. El único organismo en el Ecuador que reconoce al I es el (SECAP, 2018) que ofrece cursos módulares que permiten obtener una certificación legal de Apicultor, pero estos conocimientos no son suficientes para poder hacer frente a todos los problemas a los que se enfrentan los productores de miel.

\subsection{Déficit en la producción de miel}

"En el país existe un déficit de producción de miel. Existen dos sistemas de producción de miel de abeja, diferenciados por el nivel de tecnología utilizado. Estos son: sistema de producción familiar y sistema de producción semitecnificado." (El Telégrafo, 2018)

La falta de tecnificación de los apicultores tiene una relación directa con la producción de la miel. Según una publicación del (Diario El Mercurio Cuenca, 2019) “El Ecuador necesita aproximadamente 600 toneladas de miel de abeja al año, de las cuales solo 200 son producidas en el país y el resto es inmportsdo del mercado argentino, mexicano y chino..."

Como se puede observar en la tabla 2 el promedio por año de producción de miel en la provincia es de 20 kilogramos y est e valor no ha tenido una mayor variación en el tiempo. Según el MAGAP la producción por colmena a nivel nacional anual es de 10,2 kilos al año, el cual es muy bajo si se considera que debería de rondar los 25 a 30 kilogramos. Esta realidad también aplica para la provincia del Guayas la cual, según encuestas realizadas, mantienen un promedio de 10 kilos al año de miel de abeja, que puede variar dependiendo de la temporada y de la cantidad de alimentos que tengas las abejas a disposición.

Entre los apicultores encuestados claramente se aprecia que son pocos los que sobresalen con una producción de $14700 \mathrm{~kg}$ de miel aproximados en el transcurso de los 5 años estudiados, lo que demuestra que en efecto el sector cuenta con pequeños apicultores que en su gran mayoría no poseen más de 10 colmenas.

Resulta alarmante que a pesar de que en la provincia hay apicultores que cuentan con más de 20 años en el sector apícola no han logrado tecnificarse y mejorar sus prácticas en el proceso productivo. No obstante, hay nuevos integrantes, como se puede apreciar en la tabla 2 hay alrededor de 8 apicultores que llevan menos de 5 años en la actividad y han logrado mantener su producción constante.

Además el $47 \%$ de los apicultores no cuentan con sala de extracción, así como con equipos para llevar a cabo su producción, no obstante, el 53\% restantes cuentan con los implementos necesarios para llevar a cabo la 
actividad, por ende, realizan el proceso de manera independiente. Cabe recalcar que los empresarios optan muchas veces por alquilar los equipos entre colegas.

Tabla $\mathbf{N} 2$

Producción Anual media de miel en la provincia del Guayas en kg

Producción anual media en kg de la miel de abeja

\begin{tabular}{|c|c|c|c|c|c|}
\hline & & & Años & & \\
\hline $\mathbf{N}$ & 2015 & 2016 & 2017 & 2018 & 2019 \\
\hline 1 & 1700 & 1600 & 1800 & 2000 & 2100 \\
\hline 2 & 320 & 330 & 350 & 320 & 320 \\
\hline 3 & 160 & 175 & 180 & 150 & 200 \\
\hline 4 & 2500 & 2400 & 2400 & 2200 & 2200 \\
\hline 5 & 0 & 560 & 530 & 500 & 480 \\
\hline 6 & 180 & 180 & 150 & 120 & 120 \\
\hline 7 & 0 & 0 & 90 & 70 & 70 \\
\hline 8 & 420 & 410 & 400 & 430 & 420 \\
\hline 9 & 0 & 0 & 0 & 350 & 350 \\
\hline 10 & 1680 & 1600 & 1400 & 1500 & 1300 \\
\hline 11 & 270 & 260 & 280 & 300 & 300 \\
\hline 12 & 200 & 190 & 170 & 175 & 180 \\
\hline 13 & 400 & 390 & 400 & 400 & 420 \\
\hline 14 & 0 & 100 & 90 & 100 & 100 \\
\hline 15 & 340 & 330 & 300 & 300 & 310 \\
\hline 16 & 1300 & 1300 & 1200 & 1000 & 1000 \\
\hline 17 & 380 & 400 & 380 & 350 & 350 \\
\hline 18 & 260 & 220 & 240 & 240 & 220 \\
\hline 19 & 0 & 100 & 120 & 90 & 90 \\
\hline 20 & 3900 & 3800 & 3700 & 3600 & 3600 \\
\hline 21 & 0 & 0 & 0 & 20 & 20 \\
\hline 22 & 260 & 240 & 220 & 250 & 250 \\
\hline 23 & 1600 & 1400 & 1300 & 1100 & 1100 \\
\hline 24 & 180 & 150 & 140 & 130 & 150 \\
\hline 25 & 0 & 0 & 40 & 30 & 30 \\
\hline 26 & 0 & 80 & 75 & 60 & 80 \\
\hline 27 & 300 & 350 & 280 & 240 & 220 \\
\hline 28 & 220 & 200 & 200 & 180 & 160 \\
\hline 29 & 1500 & 1400 & 1500 & 1300 & 1300 \\
\hline 30 & 200 & 180 & 150 & 144 & 120 \\
\hline 31 & 1500 & 1300 & 1260 & 1280 & 1200 \\
\hline 32 & 2600 & 2600 & 2400 & 2200 & 2200 \\
\hline Total & 22.370 & 22.245 & 21.745 & 21.129 & 20.960 \\
\hline Toneladas & 22,037 & 22,025 & 22,075 & 21,013 & 20,10 \\
\hline
\end{tabular}

Elaborado porautores

\subsection{Falta de presencia de Apicultores Asociados a un gremio en la provincia del Guayas}

Para el sector de la apicultura la presencia de las Asociaciones es muy significativo ya gran parte del sector productor está distribuido en pequeños y medianos apicultores y se hace casi imposible competir en el mercado debido a los costos que los grandes apicultores y empresas extranjeras dedicados a la miel de abeja manejan, los cuales son mucho más inferiores que el de los apicultores pequeños y medianos, comerciar por cuenta propia para un apicultor pequeño no es muy beneficioso si es que se quiere prosperar como apicultor y vender en un tiempo más breve la totalidad de su miel. 
La provincia de Pichincha es la que más asociaciones tiene en todo el país con 4 asociaciones, la Asociación de Apicultores de Pichincha (ADAPI) tiene 82 apicultores integrantes y genera un promedio de 35 kilos de miel por colmena, solo ésta Asociación tiene un mayor número de apicultores que toda la provincia, ya que, según el catastro emitido por el MAGAP en 2018 solo existe 43 Apicultores en la provincia del Guayas.

Hay dos Asociaciones apícolas una que entro en funcionamiento el 18 de julio del 2017 Asociación de Trabajadores Apícolas Autónomos Apicultores del Ecuador con 22 Apicultores y que tiene registrada una marca comercial "Colmena Reina" con miras a comercializar a mayor escala. cabe revelar que esta apenas contaba en el 2017 con 15 colmenas lo cual no se contrasta con otras grandes Asociaciones como ADAPI que manipula en promedio 5 colmenas por socio.

Es tal la falta de integración de los apicultores que muchos se ven en desventaja a la hora de vender sus productos estos se toman más de un mes, en algunos casos dos o hasta tres meses para poder vender su stock de una cosecha, en algunos casos cuando ya se ha explotado la segunda cosecha aún se tiene almacenada parte del stock de la primera cosecha. existen algunas asociaciones en la provincia del Guayas, pero no están registradas como tal en la SEPPS (Superintendencia de Economía Popular y Solidaria) por lo cual es necesario la integración y legalización de estas asociaciones para que puedan funcionar de una mejor manera una de estas es la asociación de Apicultores autónomos de la provincia del Guayas.

Figura 2

Porcentajes de apicultores pertenecientes a asociaciones

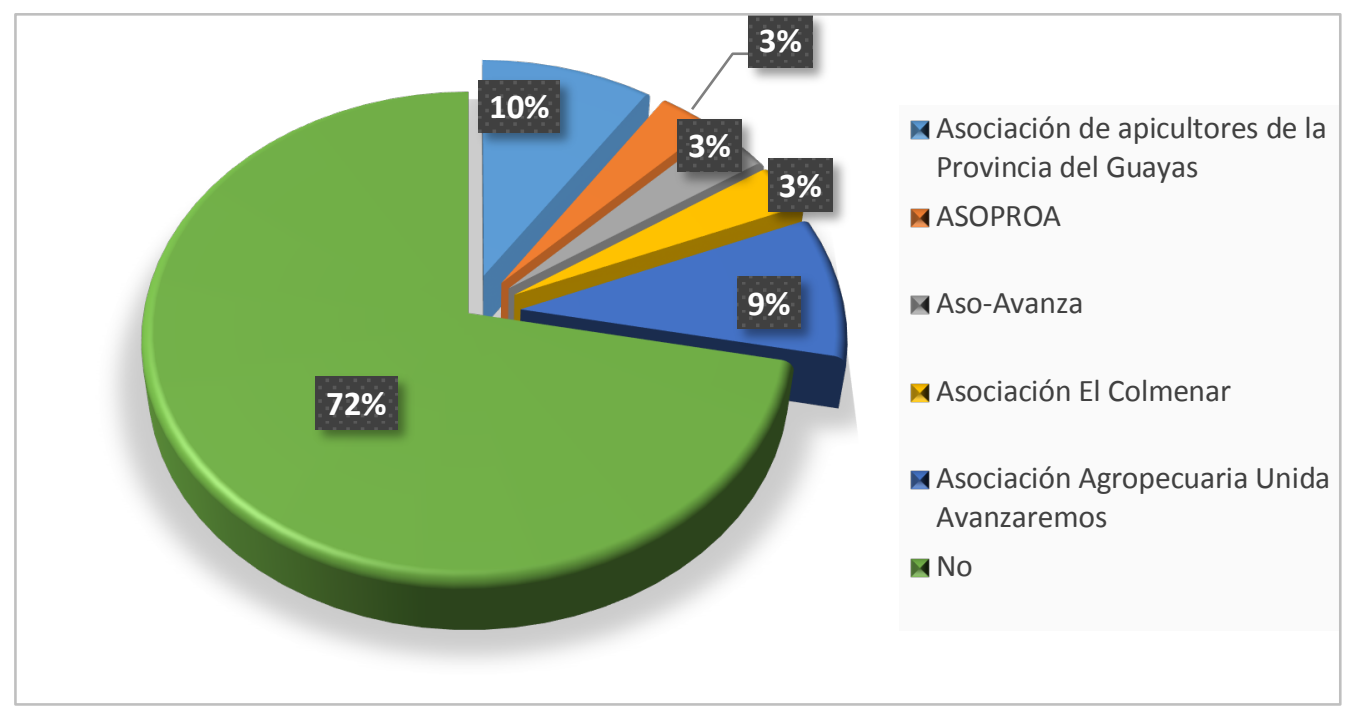

Fuente. Los autores

\subsection{Comparación de precios miel industrial y miel natural}

Después de haber hecho un amplio análisis respecto al número de apicultores que son parte de un gremio y de los beneficios que estos conlleva, se puede estudiar la manera de cómo llega el productor pequeño a competir solo en el mercado contra las mieles importadas más baratas ya sea porque su mano de obra es más flexible o porque tienen economías de escala o aquellas mieles que son adulteradas que abundan el mercado y que son producto de la mezcla entre miel natural y otros aditivos como azucares y jarabe de caña así como fructosa o la elevan a temperaturas muy elevadas para retardar su cristalización con el fin de mantenerla más tiempo en stock. Existe una brecha muy pequeña entre la miel industrial y la miel adulterada, aunque ambas tienen ciertas diferencias, pero la creación de estas dos tiene un mismo fin, abaratar costos y hacer que el producto genere más réditos. 
En la actualidad es difícil saber la diferencia entre la miel adulterada y la miel natural ya que si el consumidor va y compra miel en el mercado esta dice natural, pero en realidad no lo son o no en su totalidad, existen empresas que se dedican a la adulteración de la miel al momento de producirlas y embasarlas, pero como se da este fraude. Según la UE en la (Directiva del Consejo EUR, 2002) relacionada a la miel se permite etiquetar como miel de abeja aun si el envase solo contiene el $1 \%$ de miel natural y el $99 \%$ restantes son aditivos, estas pueden llegar a Ecuador o cualquier país destino como miel natural y los son pero solo el 1\%.,castigando así a la industria nacional.

Al año alrededor de 400 toneladas de miel de abeja son importadas desde Argentina, México y China, todo este stock llega a los principales supermercados a precios muy bajo

Tabla 3

Precio de venta entre la miel de apicultores y la miel industrial

\begin{tabular}{|c|c|c|c|}
\hline Producto & $\begin{array}{l}\text { Precio medio } \\
\text { Apicultor } \\
\text { (\$/kg) }\end{array}$ & $\begin{array}{c}\text { Precio medio } \\
\text { Industrial } \\
(\$ / \mathbf{k g})\end{array}$ & $\begin{array}{l}\text { Diferencia } \\
(\$ / \mathbf{k g})\end{array}$ \\
\hline Miel de abeja & $\$ 20$ & $\$ 10$ & $\$ 10$ \\
\hline
\end{tabular}

Información tomada del estudio de mercado. Elaborado por autores

Como se puede observar en la tabla 3 la diferencia entre los precios que se venden en los supermercados y los que se venden por medio de los apicultores es de \$10 dólares pero los precios medios que manejan los apicultores son de alrededor de \$20 dólares el kg según el catastro 2018 que maneja el MAGAP, un apicultor pequeño como se puede observar en la tabla 1, tiene entre 1-50 colmenas en teoría es lo que se maneja, pero la realidad es otra, muchos apicultores pequeños manejan entre 1 a 4 colmenas en promedio lo cual no es muy rentable.

Según entrevista con el Ing. José Baidal para que la apicultura sea rentable el apicultor tiene que manejar como promedio 10 colmenas, aquellos que manejan menos colmenas están condenados a desaparecer al largo plazo, si un apicultor maneja menos de 10 colmenas este no llega a cubrir sus costes de producción y el precio del producto final por ende ajustándose a los costos será mucho mas alto que el resto de su competencia directa, es así que en algunos casos se pueden ver diferencias entre algunos pequeños apicultores en el precio.

Estos apicultores suelen vender el kilo de miel en $\$ 12$ dólares otros en $\$ 15$ y así sucesivamente, es decir a mayor producción menor será el precio final y mayor será la oportunidad de competir en el mercado.

\subsection{Baja oferta de mieles locales en el Mercado}

Existe una gran variedad de mieles, así como de marcas en la provincia del Guayas al igual que muchos supermercados donde los expiden, para poder hacer un análisis respecto de la comercialización se procedió a realizar un trabajo de campo para poder identificar qué tipo de mieles existían en las diferentes perchas de los supermercados, hay gran cantidad de marcas provenientes de otras provincias en especial de la provincia del Pichincha, en la provincia del Guayas se encontraron marcas pero estas no eran tantas como las de otras provincias. 
Tabla 4

Marcas de miel disponibles en el mercado

originarios de otras provincias

\begin{tabular}{lccc}
\hline \multicolumn{1}{c}{ Marcas } & Supermercados & Provincias & Envases \\
\hline La abejita & Mi comisariato/tía & pichincha & $320 \mathrm{~g}$ \\
Pure Honey & Mi comisariato/super aki & Pichincha & $450 \mathrm{~g}$ \\
Maya & Tía & Pichincha & $320 \mathrm{~g}$ \\
Asoprame & Tía & Pichincha & $350 \mathrm{~g}$ \\
La obrera & Aví Fernández & Manabí & $300 \mathrm{~g}$ \\
La colmena & Tía & Azuay & $300 \mathrm{~g}$ \\
Schullo & aki/supermaxi/comisariato & Pichincha & $330 \mathrm{~g}$ \\
Aki & Super aki & Pichincha & $330 \mathrm{~g}$ \\
La bachita & Super aki & Pichincha & $300 \mathrm{~g}$ \\
Trébol & Mi comisariato & Pichincha & $300 \mathrm{~g}$ \\
Supermaxi & Supermaxi & Pichincha & $620 \mathrm{~g}$ \\
Le chateau & Supermaxi & Pichincha & $300 \mathrm{~g}$ \\
La Mega miel & Supermaxi & Pichincha & $625 \mathrm{~g}$ \\
La pradera & Supermaxi & Pichincha & $320 \mathrm{~g}$ \\
\hline
\end{tabular}

Información tomada del estudio de mercado. Elaborado por autores

Como se puede observar en la tabla N 4 las marcas de la provincia del Pichincha son las que tienen mayor presencia como antes se mencionó, son 12 las marcas de miel de esta provincia y se distribuyen desde los supermercados del mi comisariato, super aki, tía, supermaxi, existe una marca en la provincia de Manabí y 1 en la provincia del Azuay, algunos supermercados, debido a que no existen tantos distribuidores en el mercado nacional y a la poca oferta que existe en el país por el producto algunos supermercados se dedican a la producción de sus propias mieles tal es el caso del supermercado super aki que produce su propia marca de miel Aki la cual se puede encontrar en diferentes tamaños, supermaxi también produce su propia miel.

Tabla 5

Marcas producidas en la provincia

\begin{tabular}{lcl}
\hline Marcas & Supermercados & Envases \\
\hline Bee happy & Supermaxi/comisariato & $630 \mathrm{~g}$ \\
Superba & comisariato & $600 \mathrm{~g}$ \\
Reconforte & Supermaxi & $500 \mathrm{~g}$ \\
Abemiel & Supermaxi & $300 \mathrm{~g}$ \\
\hline
\end{tabular}

Información tomada del estudio de mercado. Elaborado por autores

Como se puede observar en la tabla 5 las marcas no son extensas en la provincia y 2 marcas de las 4 son marcas producidas por la misma empresa, los hermanos Lecaro, se podría decir que no existen productores locales que cubran la demanda existente en la provincia, ésta está abandonada, por parte de la empresa privada y del gobierno.

Según en la encuesta realizada por los autores de este trabajo de investigación cerca del $24 \%$ de la oferta de miel es sin etiquetado, es decir miel que se vende en forma directa al consumidor, la misma no está regulada, no cuenta con registro sanitario y con normas de calidad, mas del $80 \%$ de los consumidores prefieren comprar miel 
en forma directa al productor que comprarla en un supermercado debido al miedo que existe a que este tipo de miel sea adulterada o no sea pura.

\subsection{Baja práctica de la trashumancia en la provincia}

La base para la agricultura es la apicultura ese sería el termino correcto a utilizar para poder asociar los dos sectores, existen muchos problemas en el sector agricultor ecuatoriano debido al abandono durante años por parte de las autoridades pertinentes, muchos agricultores se quejan por la baja productividad que tienen de sus productos y los altos costos que se manejan tanto en mano de obra y los insumos, por ejemplo, hasta el 17 de junio del 2020 producir una caja de banano costaba $\$ 6$ dólares mientras que el precio real de la fruta esta en $\$ 6,16$ es decir existe solo una ganancia neta de $\$ 0,16$ centavos de dólar por cada caja de banano producido, pero como hacer que los costes bajen proporcionalmente aumentando la productividad por cada hectárea de banano, se podrían utilizar nuevas tecnologías como el uso de la biotecnología lo cual ha ayudado mucho al sector en los últimos años, pero se pasa por alto el uso de las abejas para la producción agropecuaria, utilizar a las abejas para realizar la polinización de los productos agrícolas duplica o triplica su producción, aunque se puede observar que existe una relación directa entre la practica de la trashumancia y la producción agrícola esta no se practica en la provincia del Guayas.

Figura 3

Porcentajes de apicultores que realizan trashumancia

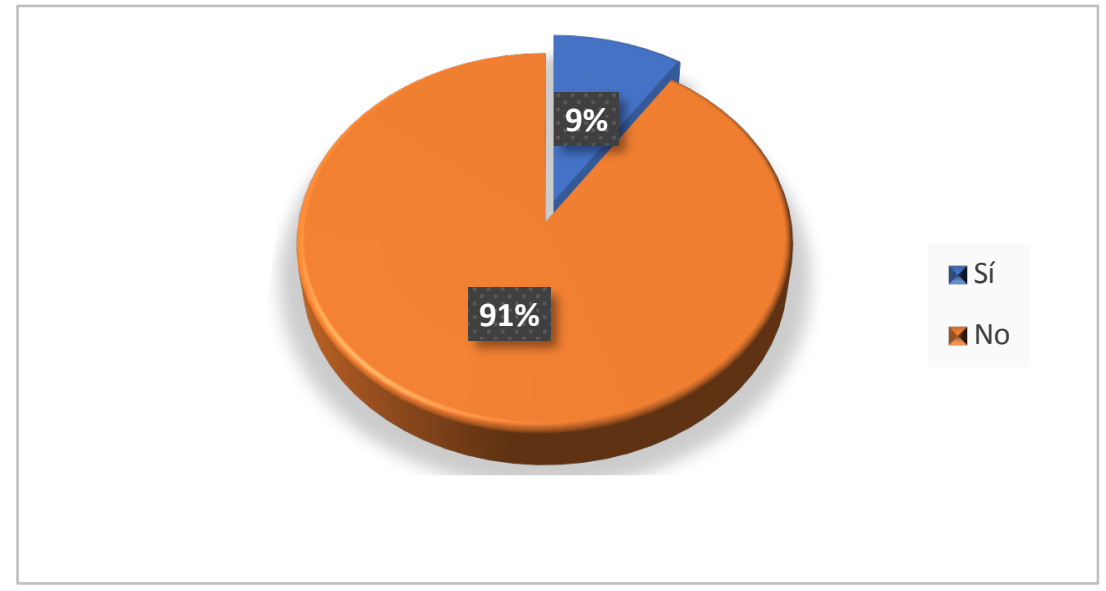

Como se puede observar en la figura 3 los apicultores que realizan la trashumancia en la provincia del Guayas es apenas $9 \%$, el $91 \%$ restante no la práctica, esto es sin duda debido a la baja demanda por medio de este servicio en la provincia por parte de los agricultores en primera instancia, pero ¿Que lleva a los agricultores a no tomar la trashumancia como alternativa a su problema de productividad? En gran parte es el desconocimiento de esta practica y de los beneficios de la apicultura a la naturaleza y el Agro, muchas veces se ve a las abejas como insectos molestos y peligrosos pero se pasa por alto que ellos son muy valiosos para el medio ambiente y para el sector alimentario, es allí donde es necesario concientizar a los agricultores de la importancia del sector apícola en sus cultivos y quien más podría impulsar esta practica si no el estado, al impulsar la trashumancia existen dos beneficios simultáneos que están directamente relacionados.

Por un lado, las abejas polinizan a los cultivos agrícolas esto impulsa la producción de los mismos, por otro lado, al tener mayor cantidad de alimentos las abejas producen mayor cantidad de miel por colmena, solucionando el problema de la baja productividad de los dos sectores, y también la calidad del producto ya que la polinización de las abejas crea un incremento en la calidad de los productos. 
Pero aquí surge un segundo problema por lo cual los apicultores no se atreven a dar este tipo de servicios y es el uso indiscriminado de pesticidas y plaguicidas en las plantaciones de cultivos, si las abejas están expuestas a un ambiente contaminado de plaguicidas esto hace que las abejas se desorienten y que se estresen lo cual hace que no lleguen a su colmena y mueran en el trayecto, entre otro tipo de problemas, para poder tener un beneficio mutuo es necesario trabajar en conjunto tanto el Agricultor como el Apicultor.

Figura 4

Catastro de Inspección sanitaria de explotaciones apícolas de la provincia del Guayas, 2018

\begin{tabular}{|c|c|c|c|c|c|c|c|c|c|c|c|}
\hline \multicolumn{2}{|c|}{$\begin{array}{l}\text { D MINISTERIO } \\
\text { DEAGRICULTURA } \\
\text { YGANADERIA } \\
\end{array}$} & \multicolumn{10}{|c|}{ INSPECCIÓN SANITARIA DE EXPLOTACIONES APÍCOLAS } \\
\hline $\begin{array}{l}\text { FORMULARIO } \\
\mathrm{N}^{\circ}\end{array}$ & PROVINCIA & CANTON & PARROQUIA & NOMBRE EXPLOTACION & MIEL & POLEN & $\begin{array}{l}\text { PRODUCCION DE } \\
\text { MATERIAL VIVO }\end{array}$ & JALEA REAL & \begin{tabular}{|l} 
TOTAL \\
COLMENAS
\end{tabular} & $\begin{array}{l}\text { MOTIVO } \\
\text { INSPECCIÓN }\end{array}$ & NOMBRE TECNICO AGROCALIDAD \\
\hline & 1 GUAYAS & NOBOL & NOBOL & APIARIO PETRILLO & 1 & 0 & 0 & 0 & & \begin{tabular}{l|l}
7 & CATASTRO \\
\end{tabular} & Mvz. María Nicole Vanegas \\
\hline & 2 GUAYAS & GUAYAQUIL & TARQUI & PREFECTURA GUAYAS & 1 & 0 & 0 & 0 & & \begin{tabular}{|l|l|}
3 & CATASTRO \\
\end{tabular} & Mvz. María Nicole Vanegas \\
\hline & 3 GUAYAS & PEDRO CARBO & SABANILLA & DAULAR & 1 & 0 & 0 & 0 & 90 & 0 CATASTRO & Mvz. María Nicole Vanegas \\
\hline & 4 GUAYAS & ALFREDO BAQ & JUJAN & DUANI & 1 & 0 & 0 & 0 & 37 & $\begin{array}{l}7 \text { CATASTRO } \\
\end{array}$ & Mvz. María Nicole Vanegas \\
\hline & 5 GUAYAS & ALFREDO BAQ & LUUJAN & & 1 & 0 & 0 & 0 & & 5 CATASTRO & Mvz. María Nicole Vanegas \\
\hline & 6 GUAYAS & ALFREDO BAQ & LUUJAN & 0 & 1 & 0 & 0 & 0 & 15 & .5 CATASTRO & Mvz. María Nicole Vanegas \\
\hline & 7 GUAYAS & ALFREDO BAQ & LUUJAN & EL RESPLANDOR & 1 & 1 & 0 & 1 & 51 & 1 CATASTRO & Mvz. María Nicole Vanegas \\
\hline & 8 GUAYAS & ALFREDO BAQ & JUJAN & LAS ZANJAS & 1 & 1 & 0 & 1 & 48 & \begin{tabular}{|l|l|}
8 & CATASTRO \\
\end{tabular} & Mvz. María Nicole Vanegas \\
\hline & 9 GUAYAS & ALFREDO BAQ & JUJAN & LAS CAÑITAS & 1 & 1 & 0 & 1 & & 6 CATASTRO & Mvz. María Nicole Vanegas \\
\hline & 0 GUAYAS & ALFREDO BAQ & JUJAN & 0 & 1 & 0 & 0 & 0 & & 1 CATASTRO & Mvz. María Nicole Vanegas \\
\hline & 1 GUAYAS & ALFREDO BAQ & LJUJAN & JUAN GABRIEL MANCILLA GONZABAY & 1 & 0 & 0 & 0 & & 5 CATASTRO & Mvz. María Nicole Vanegas \\
\hline & 2 GUAYAS & ALFREDO BAQ & JUJAN & 0 & 1 & 0 & 0 & 0 & & 4 CATASTRO & Mvz. María Nicole Vanegas \\
\hline & 3 GUAYAS & YAGUACHI & VIRGEN DE FÁTIMA & 0 & 1 & 1 & 0 & 0 & 19 & 9 CATASTRO & Mvz. María Nicole Vanegas \\
\hline & 4 GUAYAS & YAGUACHI & VIRGEN DE FÁTIMA & 0 & 1 & 1 & 0 & 0 & & 6 CATASTRO & Mvz. María Nicole Vanegas \\
\hline & 5 GUAYAS & MILAGRO & MILAGRO & 0 & 1 & 0 & 0 & 0 & & 7) CATASTRO & Mvz. María Nicole Vanegas \\
\hline & 6 GUAYAS & PEDRO CARBO & VALLE DE LA VIRGEN & LA UNIÓN & 1 & 0 & 0 & 0 & & 7 CATASTRO & Mvz. María Nicole Vanegas \\
\hline & 7 GUAYAS & PEDRO CARBO & VALLE DE LA VIRGEN & LA UNIÓN & 1 & 0 & 0 & 0 & & 1 CATASTRO & Mvz. María Nicole Vanegas \\
\hline & 8 GUAYAS & PEDRO CARBO & VALLE DE LA VIRGEN & SAN JOSE & 1 & 0 & 0 & 0 & & 1 CATASTRO & Mvz. María Nicole Vanegas \\
\hline & 9 GUAYAS & PEDRO CARBO & VALLE DE LA VIRGEN & SAN JOSE & 1 & 0 & 0 & 0 & & 4 CATASTRO & Mvz. María Nicole Vanegas \\
\hline & 0 GUAYAS & ALFREDO BAQ & LUUJAN & MIGUEL & 1 & 0 & 0 & 0 & 11 & 1 CATASTRO & Mvz. María Nicole Vanegas \\
\hline & 1 GUAYAS & SAMBORONDC & ¿TARIFA & FLOR DE TARIFA & 1 & 0 & 0 & 0 & & 2 CATASTRO & Mvz. María Nicole Vanegas \\
\hline & 2 GUAYAS & DAULE & DAULE & UNIDAD EDUCATIVA GALO PLAZA & 1 & 0 & 0 & 0 & & 3 CATASTRO & Mvz. María Nicole Vanegas \\
\hline & 3 GUAYAS & NOBOL & NOBOL & APICOLA S.V & 1 & 0 & 0 & 0 & 13 & 3 CATASTRO & Mvz. María Nicole Vanegas \\
\hline & 4 GUAYAS & EL TRIUNFO & EL TRIUNFO & BIENVENIDA & 1 & 1 & 0 & 0 & 21 & 1 CATASTRO & Mvz. María Nicole Vanegas \\
\hline & 5 GUAYAS & EL TRIUNFO & EL TRIUNFO & ANIBAL GONZALEZ & 1 & 0 & 0 & 0 & 21 & 1 CATASTRO & Mvz. María Nicole Vanegas \\
\hline & 6 GUAYAS & EL TRIUNFO & EL TRIUNFO & PATRICIO VILLAMAR & 1 & 0 & 0 & 0 & & 6 CATASTRO & Mvz. María Nicole Vanegas \\
\hline & 7 GUAYAS & COLIMES & COLIMES & CARMIEL & 1 & 0 & 0 & 0 & 25 & 15 CATASTRO & Mvz. María Nicole Vanegas \\
\hline & 8 GUAYAS & COLIMES & COLIMES & PURA MIEL & 1 & 0 & 0 & 0 & & 7] CATASTRO & Mvz. María Nicole Vanegas \\
\hline & 9 GUAYAS & PEDRO CARBO & VALLE DE LA VIRGEN & EDISSON TABARES & 1 & 0 & 0 & 0 & 20 & CATASTRO & Mvz. María Nicole Vanegas \\
\hline & 0 GUAYAS & PEDRO CARBO & VALLE DE LA VIRGEN & VALERIANO MAGALLANES & 1 & 0 & 0 & 0 & & 3 CATASTRO & Mvz. María Nicole Vanegas \\
\hline & 1 GUAYAS & PEDRO CARBO & VALLE DE LA VIRGEN & EL AGAPITO & 1 & 0 & 0 & 0 & & 2 CATASTRO & Mvz. María Nicole Vanegas \\
\hline & 2 GUAYAS & PEDRO CARBO & VALLE DE LA VIRGEN & AGAPITO 1 & 1 & 0 & 0 & 0 & & 5 CATASTRO & Mvz. María Nicole Vanegas \\
\hline & 3 GUAYAS & PEDRO CARBO & VALLE DE LA VIRGEN & AGAPITO 2 & & 0 & 0 & 0 & & 2 CATASTRO & Mvz. María Nicole Vanegas \\
\hline & 4 GUAYAS & GUAYAQUIL & CHONGON & FINCA LOLITA & 1 & 1 & 0 & 0 & & 3 CATASTRO & Mvz. María Nicole Vanegas \\
\hline & 5 GUAYAS & GUAYAQUIL & CHONGON & LA ENVIDIA & 1 & 0 & 0 & 0 & & 3 CATASTRO & Mvz. María Nicole Vanegas \\
\hline & 6 GUAYAS & NARANJITO & NARANJITO & LA DANESA & 1 & 0 & 0 & 0 & 28 & 8 REQUERIDA PQ & Mvz. Gabriela Barba Astudillo \\
\hline & 7 GUAYAS & PEDRO CARBO & SABANILLA & DAULAR & 1 & 0 & 0 & 0 & 25 & 5 RUTINARIA & Mvz. Gabriela Barba Astudillo \\
\hline & 8 GUAYAS & COLIMES & COLIMES & CON MIEL & 1 & 의 & 0 & 0 & 23 & 3 RUTINARIA & Mvz. Gabriela Barba Astudillo \\
\hline & 9 GUAYAS & COLIMES & COLIMES & TRIANA & 1 & 0 & 0 & 0 & 15 & .5 RUTINARIA & Mvz. Gabriela Barba Astudillo \\
\hline & 0 GUAYAS & BALZAR & BALZAR & LAS GUAGUAS & 1 & 0 & 0 & 0 & & 8 CATASTRO & Dr. Duberlin Matheus \\
\hline & 1 GUAYAS & PALESTINA & PALESTINA & SOTOMAYOR & 1 & 0 & 0 & 0 & 17 & .7|RUTINARIA & Dr. Duberlin Matheus \\
\hline & 2 GUAYAS & JUJAN & JUJAN & EL TIGRILLO & 1 & 0 & 0 & 0 & 20 & RUTINARIA & Mvz. Gabriela Barba Astudillo \\
\hline & 3 GUAYAS & JUJAN & JUJAN & LA PALMA & 1 & 0 & 0 & 0 & 17 & .7 RUTINARIA & Mvz. Paul Chacha \\
\hline & 4 GUAYAS & DURAN & DURAN & ELOY ALFARO & 1 & 0 & 0 & 0 & 30 & RUTINARIA & Mvz. Gabriela Barba Astudillo \\
\hline & 5 GUAYAS & DURAN & DURAN & ELOY ALFARO & 1 & 0 & 0 & 0 & 25 & 5 RUTINARIA & Mvz. Gabriela Barba Astudillo \\
\hline & 6 GUAYAS & BALAO & BALAO & LA VICTORIA & 1 & 0 & 0 & 0 & & 4 CATASTRO & Mvz. Omar Calderón \\
\hline & 7 GUAYAS & NARANJAL & TAURA & LA CAROLINA & 1 & 0 & 0 & 0 & 1 & 1 REQUERIDA PO & Mvz. Gabriela Barba Astudillo \\
\hline & 8 GUAYAS & NARANJITO & NARANJITO & LA DANESA & 1 & 0 & 0 & 0 & 40 & ONEQUERIDA PQ & Mvz. Gabriela Barba Astudillo \\
\hline & 9 GUAYAS & GUAYAQUIL & PROGRESO & DON MIGUEL & 1 & 0 & 0 & 0 & 11 & 1 REQUERIDA PQ & Mvz. Omar Andrade \\
\hline
\end{tabular}

Fuente: Ministerio de Agricultura y Ganadería, 2018 


\subsection{Políticas públicas para el sector apícola}

El Ecuador tiene el potencial necesario para poder ser uno de los primeros productores de miel de abeja a nivel sudamericano, pero es uno de los que menos produce en la región apenas producen 200 toneladas de miel al año, mientras que países como Argentina producen 76,380 toneladas de miel al año, Chile produce entre 7000 a 11,000 toneladas por año y el Perú produce 3000 toneladas por promedio al año, como se puede observar Ecuador esta muy por debajo de la media de estos países en cuanto a la producción de miel de abeja, y esto se debe al abandono del sector por parte del estado, políticas publicas que se encaminen a mejorar el sector.

Uno de los puntos a mejorar es en cuanto a sacar certificaciones de calidad para los pequeños productores, ya que resulta un problema al momento de querer realizar el trámite, sin la certificación no pueden pueden acceder al libre comercio y debido a esto la distribución del producto se lo hace entre amigos y familiares como ya se lo menciono anteriormente y quienes tienen las certificaciones de calidad pagan demasiado poco a los apicultores que no la tienen por su miel.

A esto se suma que el $75 \%$ de los apicultores encuestados consideran que no reciben ayuda por parte del Estado, indican que realizan la actividad independientemente, sin embargo, el 25\% restante indican que sí reciben apoyo de entidades u organismos estatales, tales como la Prefectura del Guayas y Agrocalidad, quienes le proporcionan control de calidad en sus apiarios, además de darles charlas y capacitaciones relacionadas a la actividad y otorgarles kits de materiales u herramientas para llevar a cabo el proceso productivo.

Otro problema es las mieles falsificadas o adulteradas ya que estas son vendidas a mitad de precio lo cual hace que el apicultor no pueda competir y al no existir una ley que proteja al apicultor este pierde credibilidad ante el consumidor, por lo cual se hace necesaria la creación de una ley que permita la estandarización de precios entre los supermercados y los pequeños apicultores para que no exista especulación de precios y un mayor control de las mieles adulteradas en cuanto al etiquetado claro para que no exista un perjuicio para el consumidor.

\section{Conclusiones}

La apicultura es una actividad necesaria para la economía ecuatoriana, así como para el medio ambiente debido a que genera ingresos para las familias de los apicultores y crea una mejora en la producción de los productos agrícolas.

Se ha observado un abandono del sector por parte de organismos estatales, mismo que repercute en la baja productividad de la actividad apícola.

A pesar de que se estima una presencia de más de 120 apicultores en la provincia, a penas se registran 43 en el MAGAP 2018, lo que demuestra que no existe por parte organismos gubernamentales un debido protocolo de seguimiento e interés para la actividad, lo que dificulta conocer la realidad del sector.

Del total de apicultores encuestados el $78 \%$ son productores artesanales, mientras que el $19 \%$ son semitecnificados y solo el $3 \%$ es tecnificado, lo cual refleja los escasos conocimientos que poseen en cuánto a técnicas para preservar sus colmenas y darles el mantenimiento adecuado.

Se presencia una baja producción de miel entre los apicultores encuestados, llegando a productir cerca de 20 toneladas de miel para el año 2019, esto debido principalmente a la poca cantidad de colmenas que poseen, siendo en su mayoría un total de 10 colmenas.

De los encuestados el $72 \%$ de apicultores no pertenecen a una asociación como tal, lo que repercute en un ostáculo para el ingreso de sus productos a los supermercados, ya que de forma independiente su producción no les da para cubrir la demanda de miel que éstos solicitan. 
Mediante el estudio de mercado se pudo observar que en los supermercados sólo 4 marcas de miel de abeja son locales, el resto de marcas ofertadas proceden de otras provincias como Pichincha. Por lo tanto, no existen productores locales que cubran la demanda existente en la provincia.

En la provincia sólo el $9 \%$ de apicultores practica la trashumancia, lo que indica un total desconocimiento de la actividad y un desaprovechimiento de la misma como alternativa de producción para la agricultura.

Existe una diferencia de precios de venta entre los apicultores y mieles industrializadas de \$10 dólares lo que hace imposible que los pequeños apicultores puedan competir al momento de quererse introducir en los supermercados.

\section{Referencias bibliográficas}

Agrocalidad. (2014). Catastro Nacccional de explotaciones Apícolas. Quito: MAGAP. Recuperado el Diciembre de 2019

Diario El Mercurio Cuenca. (27 de Abril de 2019). Ecuador necesita cada año unas 600 (tl) toneladas de miel. (CSM, Ed.) El Mercurio. Recuperado el 4 de Mayo de 2020, de https://ww2.elmercurio.com.ec/2019/04/27/ecuador-necesita-cada-ano-unas-600-tl-toneladas-de-miel/

Directiva del Consejo EUR. (2002). Directiva 2001/110/CE del Consejo, de 20 de diciembre de 2001, relativa a la miel. CONSEJO DE LA UNIÓN EUROPEA, Europa. Recuperado el 3 de Febrero de 2020, de eurlex.europa.eu/legal-content/ES/TXT/HTML/?uri=CELEX:32001L0110\&from=en

El Telégrafo. (20 de Junio de 2018). En Ecuador hay 1.400 productores de miel. El Telégrafo. Recuperado el 5 de Marzo de 2020, de https://www.eltelegrafo.com.ec/noticias/economia/4/ecuador-productores-mielapicultura

Granda, R. E. (19 de Diciembre de 2017). Análisis del potencial de la actividad apícola como desarrollo socioeconómico en sectores rurales. Recuperado el 15 de Diciembre de 2019, de Proyecto de investigación USFQ: http://repositorio.usfq.edu.ec/bitstream/23000/7106/1/135301.pdf

Ivars, J. (15 de Octubre de 2015). La Tienda del Apicultor, Blog de Apicultura. Recuperado el 10 de Abril de 2020, de Curso de Apicultura completo: https://www.latiendadelapicultor.com/blog/curso-de-apiculturacompleto/

Magaña, M. A., Cortés, M. E., Barrientos, L. L., \& García, y. J. (JUN./ AGOST. de 2016). Productividad de la apicultura en México y su impacto sobre la rentabilidad. Scielo, VII(5). Recuperado el Diciembre de 2019, de Scielo: http://www.scielo.org.mx/scielo.php?script=sci_arttext\&pid=\$2007-09342016000501103

OIE. (s.f.). Organización Mundial de Sanidad Animal. Recuperado el 16 de Febrero de 2020, de Enfermedades de las abejas: https://www.oie.int/es/sanidad-animal-en-el-mundo/enfermedades-de-losanimales/enfermedades-de-las-abejas

SECAP. (18 de Mayo de 2018). Servicio Ecuatoriano De Capacitación Profesional. Recuperado el 20 de Enero de 2020, de SECAP único organismo certificador de apicultores en el Ecuador:

https://www.secap.gob.ec/secap-unico-organismo-certificador-de-apicultores-en-el-ecuador/

\section{Anexos}

\section{Modelo de encuesta de los apicultores}




\section{Formulario de encuesta para apicultores}

\section{Nivel de escolaridad}

Nula

Primaria

Secundaria

Tercer Nivel

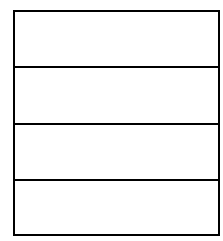

\section{Su rango de edad esta entre:}

De 18 años a 24 años

De 25 años a 34 años

De 35 años a 44 años

De 45 años a 54 años

De 55 años a 64 años

Más de 64 años

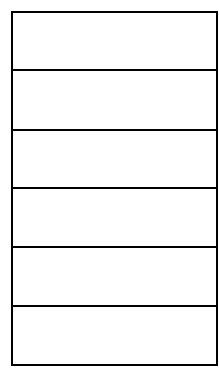

3. Indique cuál de las siguientes actividades es su principal ingreso económico Apicultura

Ganadería

Agricultura

Otro, especifique

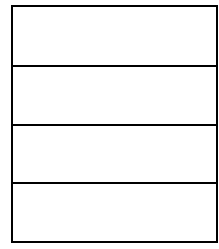

4. Ingresos mensuales que le genera el sector apícola:

De 0 a 100

De 101 hasta 200

De 201 a 300

De 301 a 400

De 401 a 500

Mayor a 500

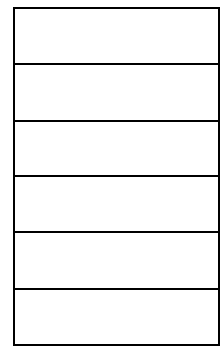

5. Indique el porcentaje de ingreso familiar que representa la Apicultura.

Menos de $25 \%$

$25-50 \%$

$50-75 \%$

Mayor 75\%

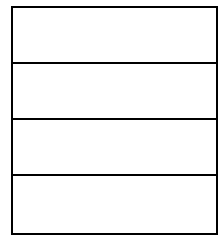

6. Número de personas que viven con usted 
Ninguna

1

2 hasta 4

5 hasta 7

Más de 7

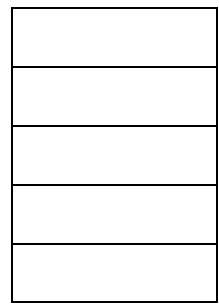

\section{Número de hijos}

Ninguno

1

2

3

4

5

Más de 5

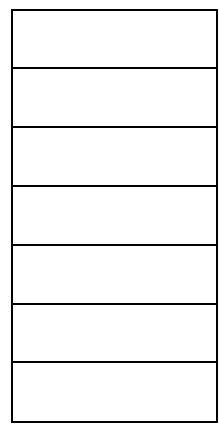

\section{Tipo de vivienda}

Casa/ villa

Rancho

Covacha

Choza

Otra (especificar)

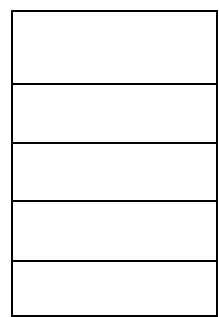

\section{9. ¿Cuenta con los siguientes servicios básicos?}
a. Energía eléctrica
b. Agua y desagüe
c. Teléfono
d. Internet

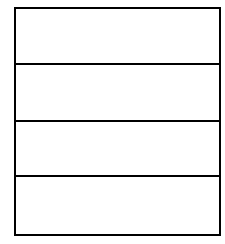

10. ¿Qué lo impulsa a realizar la actividad apícola?

Tradición familiar.

Emprendimiento e interés por la actividad.

Hobby

Otros(especifique)

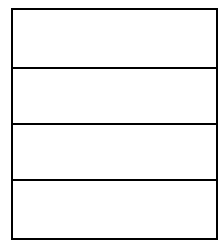

11. ¿Cuenta usted con cursos o estudios técnicos relacionados con la actividad apícola?

Si

No

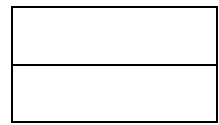


12. Cuando empezó a realizar esta actividad ¿tenía conocimiento alguno de lo que se trataba?

Si

No

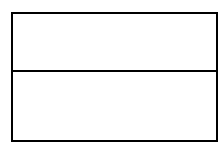

13. Indique si algún familiar trabaja con usted en la actividad Apícola.

Si. Especifique: ejemplo: Esposa,

hijo

No

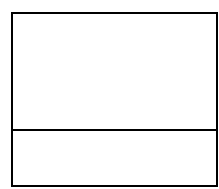

\section{4. ¿Contempla la posibilidad de optar por un relevo generacional?}

Si, mis hijos se ocuparan de las colmenas.

No, pero tengo otro tipo de sucesores.

No

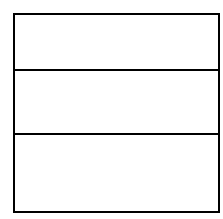

\section{Antigüedad del negocio:}

Menos de 5 años

5 a 10 años

10 a 15 años

15 a 20 años

Más de 20 años.

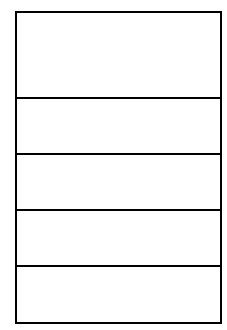

\section{Proceso productivo de la miel de abeja}

Artesanal

Semi-tecnificado

Tecnificado

\section{Tipo de negocio:}

Empresario individual

Sociedad limitada(SL)

Sociedad anónima(SA)

Asociación Economía Popular y Solidaria

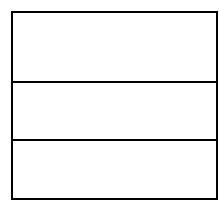

18. ¿Su apiario está cerca de su lugar de residencia?

Si

No

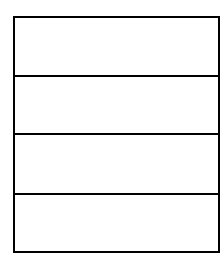


19. Bajo qué condiciones constan los terrenos en los que se encuentran los apiarios que posee.

Propio

Arriendo

Comodato

Estatal

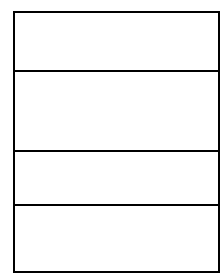

20. ¿̇ndique si cuenta usted con sala de extracción, así como instrumentos para el procesado y envasado de su propia miel?

$\mathrm{Si}$

No

Mis equipos son

prestados

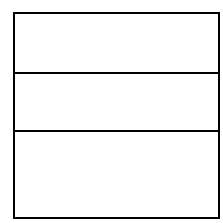

21. Indique cuanto paga por el arriendo de la sala de extracción

22. ¿Recibe usted algún tipo de subsidio o ayuda por parte del estado?

$\mathrm{Si}$

No

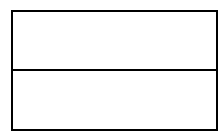

23. Indique si poseen un fácil acceso a financiamiento por parte de la banca:

Publica

Privada

Ninguna

24. ¿Considera usted que las tasas de los créditos son altas?

$\mathrm{Si}$

No

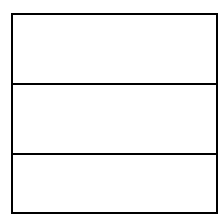

25. Usted es miembro de alguna Asociación de apicultores:

Asociación de apicultores de la provincia del

Guayas

Asociación de trabajadores apícolas Autónomos Apicultores del Ecuador.

Otra, especifique

No

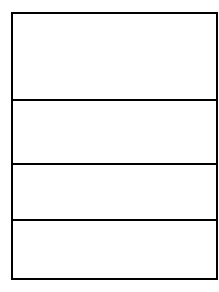

26. ¿Cuenta con una marca registrada legalmente?

$\mathrm{Si}$

No

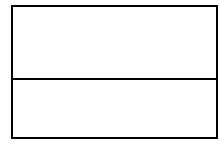


En proceso

27. Comercializa sus productos bajo una determinada marca de calidad ejemplo (INEN)

No

Si. especifique:

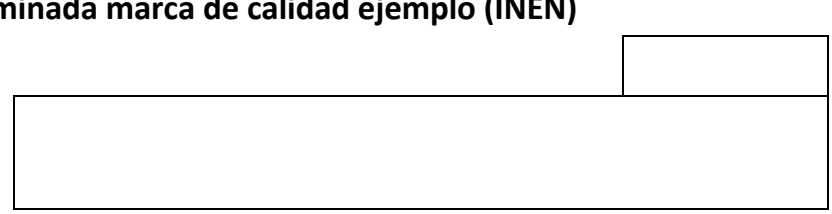

28. Realiza práctica la trashumancia (pastoreo continuo movimiento)

$\mathrm{Si}$

No

29. Locación en donde practica tranhumancia

30. ¿Qué tipo de abeja posee o usa para su actividad?

Italianas

Africanizadas

Cárnica

Negra

Ibérica

Otra

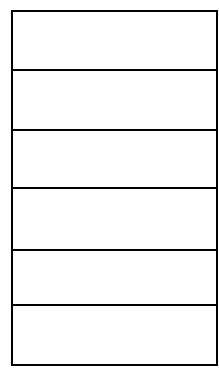

31. ¿Se ha visto afectado por las plagas y enfermedades que afectan a las abejas? En caso que su respuesta sea si, indique ¿Cuáles son esas plagas o enfermedades?

32. ¿De qué tamaño es su colmena?

Pequeña (Entre 1 a 50 colmenas)

Mediana (Entre 51 a 150 colmenas)

Grande (Mayor a 150 colmenas)

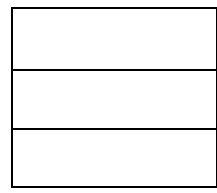

33. ¿Cuántas cosechas realiza al año? Especifique cuantos (lt, $\mathrm{kg}, \mathrm{gr}, \mathrm{t}$ ) produce por cada cosecha

1

2

3

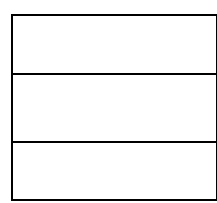

34. El número de colmenas en el año 2019:

Aumentó 
Disminuyó

35. Indique la cantidad de miel de abeja cosechada al año por colmena

$10 \mathrm{~kg}$

$20 \mathrm{~kg}$

$30 \mathrm{~kg}$

Otros

36. Indique cuál es su producción media de miel al año

\begin{tabular}{|l|l|}
\hline Año & Producción/media \\
\hline 2015 & \\
\hline 2016 & \\
\hline 2017 & \\
\hline 2018 & \\
\hline 2019 & \\
\hline
\end{tabular}

37. Aproximadamente ¿Cuál es su costo de producción?

38. Indique que otros productos comercializa en su empresa a parte de la miel de abeja. Y qué porcentaje representa de su producción.

\begin{tabular}{|l|l|l|}
\hline Productos que & $\begin{array}{l}\text { Productos de } \\
\text { produce(x) } \\
\text { producción(\%) }\end{array}$ \\
\hline Cera/velas & & \\
\hline Miel de abeja & & \\
\hline Propóleos & & \\
\hline Panales & & \\
\hline Jalea real & & \\
\hline Caramelos & & \\
\hline Línea cosmética & & \\
\hline
\end{tabular}

\section{Su producción se destina en mayor proporción a}

Comercio

Autoconsumo

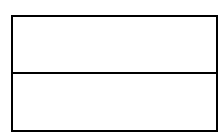

40. Indique si utiliza el apoyo de otros organismos para la venta de su miel

No, el total de mis ventas las gestiono por mi cuenta

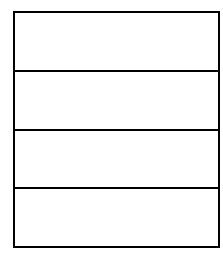


Utilizo la ayuda de intermediarios para la venta de mis productos

Gestiono mis ventas a través de una empresa diferente a la mía

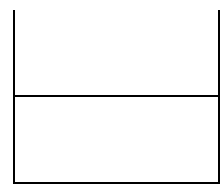

41. Indique cuales son los problemas a las que se enfrenta la actividad de la apicultura en la provincia del Guayas. (varias opciones)

Uso de pesticidas y otros productos químicos que dañan a las abejas

Costo elevado de mano de obra y del proceso

productivo

Dificultad para obtener áreas verdes actas para

la Apicultura

Precios de mercado muy bajos

Bajo nivel de tecnificación de los apicultores

Poca ayuda por parte del estado en subsidios o

programas para el desarrollo del sector

Otros, especifique

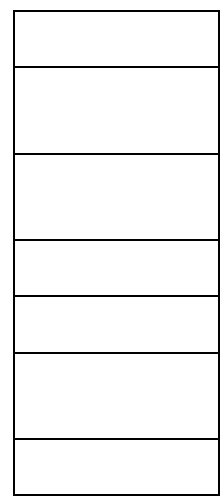

\section{La venta de su miel va dirigida al mercado (varias opciones)}

Local

Nacional

Regional

Internacional

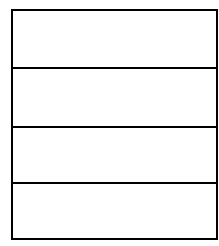

43. Indique los sitios donde comercializa la miel de abeja (varias opciones)

Supermercados

Internet

Venta directa al

consumidor

Ferias

Tiendas naturistas

Otros. especifique:

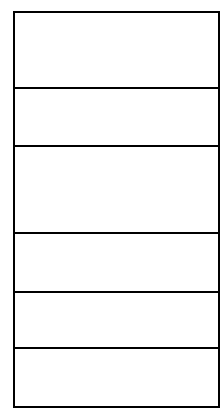

44. Indique el precio aproximado que cobra por su producto en los sitios antes mencionados

\begin{tabular}{|l|l|}
\hline $\begin{array}{l}\text { Sitios de } \\
\text { comercialización: }\end{array}$ & $\begin{array}{l}\text { Precio de venta de Miel } \\
(\$ / \mathrm{kg})\end{array}$ \\
\hline Supermercados & \\
\hline Internet directa al & \\
\hline $\begin{array}{l}\text { Venta } \\
\text { consumidor }\end{array}$ & \\
\hline Ferias & \\
\hline Tiendas naturistas & \\
\hline Otros & \\
\hline
\end{tabular}


45. El envase para la venta de su miel es de:

Envase de 100 gramos

Envase de 300 gramos

Envase de 350 gramos

Otros. especifique

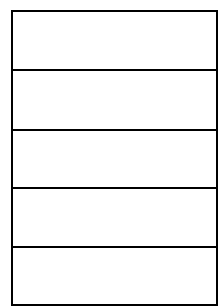

46. Aproximadamente, ¿Qué tiempo le toma vender su producción en el mercado?

\section{7. ¿Qué dificultades encuentra para vender su miel?}

48. Oferta su producto a través de alguna red social?

Facebook

Instagram

Twitter

Publicidad pagada

Volantes

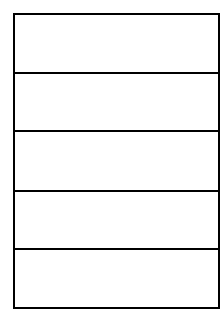

49. Indique en el siguiente cuadro el número de empleados que utiliza para su actividad apícola:

\begin{tabular}{|l|l|l|}
\hline & Hombres & Mujeres \\
\hline Fijos & & \\
\hline Eventuales & & \\
\hline
\end{tabular}

50. Indique con una $x$ cual es la importancia y beneficios que brinda la apicultura en la provincia del Guayas.

\begin{tabular}{|l|l|l|l|l|}
\hline & Sin importancia & Poco importante & Importante & $\begin{array}{l}\text { Muy } \\
\text { importante }\end{array}$ \\
\hline Generación de empleo & & & & \\
\hline $\begin{array}{l}\text { Impulsa la producción } \\
\text { de productos agrícolas }\end{array}$ & & & & \\
\hline Ayuda el ecosistema & & & & \\
\hline Otros & & & & \\
\hline
\end{tabular}


Anexo 1. Modelo de encuesta a los consumidores

\section{ENCUESTA AL CONSUMO DE LA MIEL DE ABEJA}

1. Edad

2.Sexo: Masculino

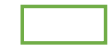

Femenino

3. Indique cuál es su nivel de estudio:

Primaria $\square$ secundaria

estudios universitarios

4.En su vida diaria:

- Cuido de mi alimentación

- Evito un alto consumo de grasas y azucares

- No me preocupa mi alimentación

5.Consume miel de abeja:
○ $\mathrm{Si}$
○ No

\section{ENCASO DE QUE CONSUMA MIEL DE ABEJA PASE DIRECTAMENTE A LA PREGUNTA N.12}

\section{para no consumidores:}

6. Indique el motivo por el que no consume miel de abeja:

- No me gusta (sabor, olor, textura)

- No conozco el producto y sus beneficios

- Otros

7.Consumiría miel si conociese de sus beneficios
○ $\mathrm{Si}$
○ No
- quizás

8. Indique si conoce usted que la miel de abeja es un producto que no contiene conservante, edulcorantes
○ $\mathrm{Si}$
○ No

9. Conoce la importancia que tienen las abejas para el medio ambiente
○ Si
○ No
- Ligeramente tengo conocimiento de aquello.

10. recomendaría el producto a una persona que busque un sustituto para endulzante como el azúcar:
○ $\mathrm{Si}$
O No

11.cree usted que sería adecuada su disponibilidad en bares, restaurantes o cafeterías como una elección para un endulzante natural 
$\circ \quad \mathrm{Si}$

\section{Para consumidores:}

12. Indique cada cuanto consume miel de abeja
- Diariamente
- 2 a 3 veces por semana
- 1 vez a la semana
- 1 vez cada 15 días
- 1 vez al mes
- 1 vez al año

13. indique porque motivos consume miel de abeja
- Porque me gusta el producto (sabor, color, textura)
- Debido a sus propiedades saludables
- Debido a que es un sustituto de azúcar
- Debido a su aporte energético.
o Otros.

14. indique en que épocas del año consume la Miel
○ Invierno
- Verano
- Indistintamente de la época

\section{Sobre el producto:}

15. Indique en qué lugar compra el producto
○ Internet
- Supermercados
- Al propio productor
- Ferias
- Mercados
- Tiendas naturistas
- Otros. especifique:

16.Indique que tipo de miel consume:
- Natural
- Industrial
- Compro los dos tipos

17. Que envase de venta prefiere.
- Tarro de cristal $1 \mathrm{~kg}$
- Tarro de cristal $150 \mathrm{~g}$
- Tarro de cristal $300 \mathrm{~g}$
- Tarro de cristal $350 \mathrm{~g}$
- Otros. especifique:

18.Cree usted que la textura, y el grado de cristalización tienen una influencia en la calidad

○ Si 
No

19.Indique cuál de las opciones usa para comprobar la calidad de la miel
- Color
- Densidad
- Grado de cristalización
- Tipo de miel
o Otros

20.Indique el precio que estaría dispuesto a pagar por la miel de abeja pura/natural de un envase de $300 \mathrm{~g}$
○ $3-5 \$$
$0 \quad 5-7 \$$
○ 7-9\$
○ $9-11 \$$
○ Otro. especifique:

21.indique el precio que estaría dispuesto a pagar por una miel industrial en un envase de $300 \mathrm{~g}$.
○ $3-5 \$$
○ $5-7 \$$
○ 7-9\$
○ 9-11\$
- No me interesa adquirir el producto

22. Indique si consumiría miel producida en el país o prefiere la producida en el exterior

Si, consumiría la miel nacional

No, consumiría la miel extranjera.

23. Indique cuál de estas marcas de miel prefiere de las que se venden en el mercado.
Miel de abeja maya

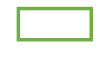
La abejita
Bachita

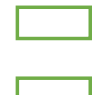
Asoprame
Grand Mother
La colmena
schullo
La obrera
Pure Honey

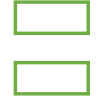
Colmena reina

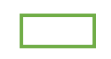
Miel de abeja Aki
otros

Anexo 2. Tabulación de resultados de encuestas a los apicultores

\begin{tabular}{|l|r|r|}
\cline { 2 - 3 } \multicolumn{1}{c|}{} & \multicolumn{2}{c|}{ Respuestas } \\
\hline Nivel de educación & $\mathrm{N}$ & \multicolumn{2}{c|}{$\%$} \\
\hline Nula & 0 & $0 \%$ \\
\hline Primaria & 8 & $25 \%$ \\
\hline Secundaria & 7 & $22 \%$ \\
\hline Tercer nivel & 17 & $53 \%$ \\
\hline
\end{tabular}


32 $100 \%$

\begin{tabular}{|l|l|r|r|}
\cline { 3 - 4 } \multicolumn{2}{c|}{} & $\mathrm{N}$ & \multicolumn{1}{|c|}{$\%$} \\
\hline \multirow{3}{*}{ Otras actividades } & Jornalero & 1 & $3 \%$ \\
\cline { 2 - 4 } & Docente & 2 & $6 \%$ \\
\cline { 2 - 4 } & Laboro en empresa & 4 & $13 \%$ \\
\cline { 2 - 4 } & Industria & 1 & $3 \%$ \\
\hline \multicolumn{2}{|l|}{} & $\mathbf{8}$ & $\mathbf{2 5 \%}$ \\
\hline
\end{tabular}

\begin{tabular}{|c|c|c|}
\hline & \multicolumn{2}{|c|}{ Ingreso mensual apicultura } \\
\hline Opción de Respuesta & $\mathrm{N}$ & $\%$ \\
\hline De 0 a 100 & 8 & $25 \%$ \\
\hline De 101 a 200 & 1 & $3 \%$ \\
\hline De 201 a 300 & 4 & $13 \%$ \\
\hline De 301 a 400 & 2 & $6 \%$ \\
\hline De 401 a 500 & 1 & $3 \%$ \\
\hline Más de 500 & 16 & $50 \%$ \\
\hline & 32 & $100 \%$ \\
\hline
\end{tabular}

\begin{tabular}{|l|r|r|}
\cline { 2 - 3 } \multicolumn{1}{c|}{} & \multicolumn{2}{c|}{ familia que convive } \\
\hline Opción de Respuesta & $\mathrm{N}$ & \multicolumn{2}{c|}{$\%$} \\
\hline Ninguna & 0 & $0 \%$ \\
\hline 1 & 1 & $3 \%$ \\
\hline 2 a 4 & 16 & $50 \%$ \\
\hline 5 a 7 & 14 & $44 \%$ \\
\hline Más de 7 & 1 & $3 \%$ \\
\hline & 32 & $\mathbf{1 0 0} \%$ \\
\hline
\end{tabular}

\begin{tabular}{|c|r|r|}
\cline { 2 - 3 } \multicolumn{1}{c|}{} & \multicolumn{2}{c|}{ Número de hijos } \\
\hline Opción de Respuesta & $\mathrm{N}$ & $\%$ \\
\hline 0 & 4 & $12 \%$ \\
\hline 1 & 12 & $38 \%$ \\
\hline 2 & 7 & $22 \%$ \\
\hline
\end{tabular}




\begin{tabular}{|c|r|r|}
\hline 3 & 5 & $16 \%$ \\
\hline 4 & 1 & $3 \%$ \\
\hline 5 & 2 & $6 \%$ \\
\hline Más de 5 & 1 & $3 \%$ \\
\hline \multirow{2}{*}{} & 32 & $100 \%$ \\
\hline
\end{tabular}

\begin{tabular}{|l|r|r|}
\cline { 2 - 3 } \multicolumn{1}{c|}{} & \multicolumn{2}{c|}{ Respuestas } \\
\hline Opción de Respuesta & $\mathrm{N}$ & \multicolumn{1}{c|}{$\%$} \\
\hline Casa/villa & 30 & $94 \%$ \\
\hline Rancho & 0 & $0 \%$ \\
\hline Covacha & 0 & $0 \%$ \\
\hline Choza & 0 & $0 \%$ \\
\hline Apartamento & 1 & $3 \%$ \\
\hline Hacienda & 1 & $3 \%$ \\
\hline \multirow{2}{*}{} & $\mathbf{3 2}$ & $\mathbf{1 0 0 \%}$ \\
\hline
\end{tabular}

\begin{tabular}{|l|r|r|}
\cline { 2 - 3 } \multicolumn{1}{c|}{} & \multicolumn{2}{c|}{ Motivo de emprendimiento } \\
\hline Opción de Respuesta & $\mathrm{N}$ & \multicolumn{1}{c|}{$\%$} \\
\hline Tradición familiar & 8 & $25 \%$ \\
\hline Emprendimiento e interés por la actividad & 20 & $63 \%$ \\
\hline Hobby & 9 & $28 \%$ \\
\hline
\end{tabular}

\begin{tabular}{|l|r|r|}
\cline { 2 - 3 } \multicolumn{1}{c|}{} & \multicolumn{2}{c|}{ Con quien realiza la actividad } \\
\hline Opción de Respuesta & $\mathrm{N}$ & \multicolumn{2}{c|}{$\%$} \\
\hline Esposa & 8 & $15 \%$ \\
\hline Hijos & 11 & $20 \%$ \\
\hline Hermano & 7 & $13 \%$ \\
\hline Primo & 1 & $2 \%$ \\
\hline Nuera & 1 & $2 \%$ \\
\hline Cuñado & 1 & $2 \%$ \\
\hline
\end{tabular}




\begin{tabular}{|c|c|c|c|c|}
\hline \multirow{2}{*}{ Opción de Respuesta } & & \multicolumn{3}{|c|}{ Respuestas } \\
\hline & & $\mathrm{N}$ & \multicolumn{2}{|l|}{$\%$} \\
\hline Sí, mis hijos se ocuparán de las colmenas & enas & 17 & \multicolumn{2}{|r|}{$53 \%$} \\
\hline \multicolumn{2}{|l|}{ No, pero tengo otro tipo de sucesores } & 7 & \multicolumn{2}{|r|}{$22 \%$} \\
\hline \multirow[t]{3}{*}{ No } & & 8 & & $25 \%$ \\
\hline & & 32 & & $100 \%$ \\
\hline & \multicolumn{3}{|c|}{ Cuenta con equipo para la apicultura } & \\
\hline Opción de Respuesta & $\mathrm{N}$ & \multicolumn{2}{|c|}{$\%$} & \\
\hline Si & 13 & & $41 \%$ & \\
\hline No & 15 & & $47 \%$ & \\
\hline \multirow[t]{2}{*}{ Mis equipos son prestados } & 4 & & $12 \%$ & \\
\hline & 32 & & $100 \%$ & \\
\hline
\end{tabular}

\begin{tabular}{|c|c|c|}
\hline \multirow[b]{2}{*}{ Opción de Respuesta } & \multicolumn{2}{|c|}{ Fácil acceso a financiamiento } \\
\hline & $\mathrm{N}$ & $\%$ \\
\hline Pública & 3 & $9 \%$ \\
\hline Privada & 1 & $3 \%$ \\
\hline \multirow[t]{2}{*}{ Ninguna } & $2 \varepsilon$ & $88 \%$ \\
\hline & 32 & $100 \%$ \\
\hline
\end{tabular}

\begin{tabular}{|l|l|r|r|}
\cline { 3 - 4 } \multicolumn{2}{c}{} & N & \multicolumn{1}{c|}{$\%$} \\
\hline \multirow{2}{*}{ Registro legal } & Registro Sanitario & 3 & $9.75 \%$ \\
\cline { 2 - 4 } & INEN & 1 & $3.25 \%$ \\
\hline \multicolumn{2}{|l}{} & 4 & $13 \%$ \\
\hline
\end{tabular}

\begin{tabular}{|l|r|r|}
\cline { 2 - 3 } \multicolumn{1}{c|}{} & \multicolumn{2}{c|}{ Tipo de abeja } \\
\hline Opción de Respuesta & $\mathrm{N}$ & \multicolumn{2}{c|}{$\%$} \\
\hline Italiana & 1 & $3 \%$ \\
\hline Africanizada & 16 & $50 \%$ \\
\hline Cárnica & 0 & $0 \%$ \\
\hline Negra & 0 & $0 \%$ \\
\hline Ibérica & 0 & $0 \%$ \\
\hline Híbrida & 13 & $41 \%$ \\
\hline
\end{tabular}




\begin{tabular}{|l|r|r|} 
Melífera & 2 & $6 \%$ \\
\hline & 32 & $100 \%$ \\
\hline
\end{tabular}

\begin{tabular}{|l|r|r|}
\cline { 2 - 3 } \multicolumn{1}{c|}{} & \multicolumn{3}{c|}{ Colmenas durante el 2019 } \\
\hline Opción de Respuesta & $\mathrm{N}$ & \multicolumn{1}{c|}{$\%$} \\
\hline Aumentó & 7 & $22 \%$ \\
\hline Disminuyó & 8 & $25 \%$ \\
\hline Se mantuvo & 17 & $53 \%$ \\
\hline \multirow{2}{*}{} & 32 & $100 \%$ \\
\hline
\end{tabular}

\begin{tabular}{|l|c|r|}
\hline Costo de producción anual & $\mathrm{N}$ & \multicolumn{2}{|c|}{$\%$} \\
\hline$\$ 10-\$ 40$ & 8 & $25 \%$ \\
\hline$\$ 60-\$ 120$ & 9 & $28 \%$ \\
\hline$\$ 150-\$ 250$ & 5 & $16 \%$ \\
\hline$\$ 300-\$ 550$ & 4 & $12 \%$ \\
\hline Más de \$1000 & 2 & $6 \%$ \\
\hline$\$ 0$ & 4 & $13 \%$ \\
\hline
\end{tabular}

\begin{tabular}{|c|c|c|c|}
\hline \multirow[b]{2}{*}{ Opción de Respuesta } & \multicolumn{2}{|c|}{ Temporadas de mayor venta } & \\
\hline & Invierno & Verano & $\%$ \\
\hline Aumenta & 0 & 32 & $100 \%$ \\
\hline Disminuye & 32 & 0 & $100 \%$ \\
\hline
\end{tabular}

\begin{tabular}{|c|c|c|}
\hline \multirow[b]{2}{*}{ Opción de Respuesta } & \multicolumn{2}{|c|}{ Destino de las ventas } \\
\hline & $\mathrm{N}$ & $\%$ \\
\hline Local & 29 & $91 \%$ \\
\hline Regional & 2 & $6 \%$ \\
\hline Nacional & 1 & $3 \%$ \\
\hline \multirow[t]{2}{*}{ Internacional } & 0 & $0 \%$ \\
\hline & 32 & $100 \%$ \\
\hline
\end{tabular}




\begin{tabular}{|c|c|c|}
\hline & \multicolumn{2}{|c|}{ Tiempo de venta del producto } \\
\hline Opción de Respuesta & $\mathrm{N}$ & $\%$ \\
\hline Inmediata & 11 & $34 \%$ \\
\hline $1 \mathrm{mes}$ & 4 & $13 \%$ \\
\hline 2 meses & 4 & $12 \%$ \\
\hline 3 meses & 5 & $16 \%$ \\
\hline Durante todo el año & 6 & $19 \%$ \\
\hline \multirow[t]{2}{*}{ No la vendo } & 2 & $6 \%$ \\
\hline & 32 & $100 \%$ \\
\hline
\end{tabular}

\begin{tabular}{|l|r|r|}
\cline { 2 - 3 } \multicolumn{1}{c|}{} & \multicolumn{2}{c|}{ Publicidad por redes sociales } \\
\hline Opción de Respuesta & $\mathrm{N}$ & \multicolumn{1}{c|}{$\%$} \\
\hline Facebook & 9 & $22 \%$ \\
\hline Instagram & 5 & $12 \%$ \\
\hline Twitter & 3 & $7 \%$ \\
\hline Publicidad pagada & 1 & $2 \%$ \\
\hline Volantes & 1 & $2 \%$ \\
\hline No & 23 & $55 \%$ \\
\hline
\end{tabular}

\begin{tabular}{|l|r|r|r|r|}
\cline { 2 - 6 } \multicolumn{1}{c|}{} & \multicolumn{4}{c|}{ Importancia de la apicultura } \\
\hline Opción de Respuesta & Sin importancia & $\begin{array}{c}\text { Poco } \\
\text { importante }\end{array}$ & Importante & $\begin{array}{l}\text { Muy } \\
\text { importante }\end{array}$ \\
\hline Genera empleo & 2 & 4 & 2 & 24 \\
\hline $\begin{array}{l}\text { Impulsa la producción } \\
\text { productos agrícolas }\end{array}$ & 0 & 0 & 4 & 28 \\
\hline Beneficia el ecosistema & 1 & 1 & 2 & 28 \\
\hline
\end{tabular}

\begin{tabular}{|l|r|r|}
\cline { 2 - 3 } \multicolumn{1}{c|}{} & \multicolumn{2}{c|}{ Respuestas } \\
\hline Servicios básicos & $\mathrm{N}$ & \multicolumn{1}{c|}{$\%$} \\
\hline Energía eléctrica & 32 & $100 \%$ \\
\hline Agua y desague & 29 & $90,63 \%$ \\
\hline Teléfono & 25 & $78,13 \%$ \\
\hline Internet & 25 & $78,13 \%$ \\
\hline
\end{tabular}


Anexo 3. Tabulación de respuestas de las encuestas a los consumidores

\begin{tabular}{|l|r|r|}
\cline { 2 - 3 } \multicolumn{1}{c|}{} & \multicolumn{2}{c|}{ Consumidores por sexo } \\
\hline Opción de Respuesta & $\mathbf{N}$ & \multicolumn{2}{c|}{$\%$} \\
\hline Masculino & 146 & $38 \%$ \\
\hline Femenino & 238 & $62 \%$ \\
\hline
\end{tabular}

\begin{tabular}{|l|r|r|}
\cline { 2 - 3 } \multicolumn{1}{c|}{} & \multicolumn{2}{c|}{ Respuestas } \\
\hline Opción de Respuesta & $\mathbf{N}$ & \multicolumn{2}{c|}{$\%$} \\
\hline Primaria & 37 & $10 \%$ \\
\hline Secundaria & 139 & $36 \%$ \\
\hline Tercer nivel & 202 & $53 \%$ \\
\hline Cuarto nivel & 4 & $1 \%$ \\
\hline Ninguna & 2 & $0 \%$ \\
\hline & 384 & $\mathbf{1 0 0 \%}$ \\
\hline
\end{tabular}

\begin{tabular}{|l|r|r|}
\cline { 2 - 3 } \multicolumn{1}{c|}{} & \multicolumn{2}{c|}{ consumo de la miel de abeja } \\
\hline Opción de Respuesta & N & \multicolumn{2}{c|}{$\%$} \\
\hline Invierno & 45 & $20 \%$ \\
\hline Verano & 8 & $4 \%$ \\
\hline Indistintamente de la época & 166 & $\mathbf{7 6 \%}$ \\
\hline & $\mathbf{2 1 9}$ & $\mathbf{1 0 0 \%}$ \\
\hline
\end{tabular}

\begin{tabular}{|l|r|r|}
\cline { 2 - 3 } \multicolumn{1}{c|}{} & \multicolumn{2}{c|}{ Consumo miel nacional e importada } \\
\hline Opción de Respuesta & N & \multicolumn{1}{c|}{$\%$} \\
\hline Sí, consumo miel nacional & 201 & $92 \%$ \\
\hline No, consumo miel importada & 18 & $\mathbf{8} \%$ \\
\hline
\end{tabular}

Esta obra está bajo una Licencia Creative Commons Attribución-NoCommercial 4.0 International

\section{(cc) E EY-NC}

